\title{
A Piezoelectric Goniometer Inside a TEM Goniometer
}

\author{
W. Guan, A. Lockwood, B.J. Inkson, G. Möbus \\ Department of Engineering Materials, University of Sheffield, Sheffield S1 3JD, U.K.
}

Piezoelectric nanoactuators, which can provide extremely stable and reproducible positioning, are rapidly becoming the dominant means for position control in transmission electron microscopy (TEM). This technology links the accurate manipulation of samples at the nanometer regime with TEM's imaging resolution and therefore offers a better way to advance the understanding of the properties of nano-scale systems [1-3]. A family of piezo-drives inserted into TEM goniometers have been developed for different applications, such as nano-contacting, electrical measurement, mechanical and tribological test by indentation, as well as electron tomography which reveals the objects' physical and chemical structures in three dimensions (3D) [4-6].

In this paper, we present a $2^{\text {nd }}$ generation miniature goniometric nanomanipulation system which is fitted inside a hollowed standard specimen holder for JEOL 2000/3000 series microscopes with neither modifications to the TEM nor compromising the performance of the default TEM goniometer. The movement range of this miniaturized drive is composed of seven degrees of freedom: 3 fine translational movements (X, Y and $\mathrm{Z}$ axes) achieved by a quartered piezo tube, further coarse translational movements along all three axes, operated serially to the fine drive, and one rotational movement around the $\mathrm{X}$-axis. Both the coarse drive and the rotary drive are using a slip-stick inertial slider mechanism. The whole system and rotary drive are schematically depicted in FIG. 1. and FIG. 2. respectively. The coarse drive schematic diagram can be found in [4].

The nanomanipulation system is ideal for electron tomography as it overcomes the "missing wedge problem" by providing a full tomographic tilt range of $180^{\circ}$ in both directions and even beyond, with the tilt oriented around the TEM holder axis. The smallest angular increment is decided by a single slip-stick actuation pulse, hence it can be well controlled to be less than $0.01^{\circ}$. The maximum rotation speed is up to $30^{\circ}$ per minute. Although in different parts of the rotation circle the speed can be different due to potentially unevenly distributed load on the shaft, non-uniform surface finish of the shaft etc., using an angular displacement feedback the accuracy of which is down to $0.05^{\circ}$, we can obtain the absolute rotated angle without relying on counting drive pulses or image calibration. FIG. 3. shows the movement of the specimen holder in a $360^{\circ}$ full circle rotation, measured via a fibre optic position sensor, without the compensation from the fine or the coarse translational drive. The movement is restricted to an extremely narrow space therefore this device enables tilting of over $180^{\circ}$ in TEMs with a $2 \mathrm{~mm}$ pole piece gap.

With the fine and course translational drives, the miniaturized nanomanipulation system is not only usable for electron tomography but also capable of many other applications. The coarse drive provides independent displacement in three axes with a macroscopic travel range more than $1 \mathrm{~mm}$ and a step resolution less than 100nm, while the resolution of the fine drive, which could be addressed to sub-0.1nm steps, is ultimately limited by vibrations, dependent on specimen mount. With the second specimen mount on the TEM holder, differential rotation between two parts (using TEM goniometer for fixed specimen, and piezo-rotation for moveable specimen) is achieved. The schematic diagram and TEM image are depicted in FIG 4 and 5. The novel rotation-translation drive 
is therefore ideal for applications such as orientation-specific nanoindentation or electrical contacting experiments. This latter property defines our "goniometer inside the TEM goniometer" approach, with both goniometers applicable in parallel or in series.

We acknowledge contributions to the project by X.J. Xu, R. Gay, Y.Peng, and J.J. Wang. [7]

References

[1] E.A. Stach et al., Microsc. Microanal, 7 (2001) 507.

[2] T. Kizuka et al., Phys. Rev. B, 55 (1997) R7398-401.

[3] M.S. Bobji et al., Meas. Sci. Technol., 17 (2006) 1324.

[4] X.J. Xu et al., Microsc. Res. Tech., 71 (2008) 773.

[5] A.J. Lockwood et al., EMAG, 126 (2008) 12096.

[6] A.J. Lockwood et al., J. Phys. D, 42 (2009) 035410.

[7] This project was funded by the EPSRC Basic Technology Programme,UK, EP/G036748/1

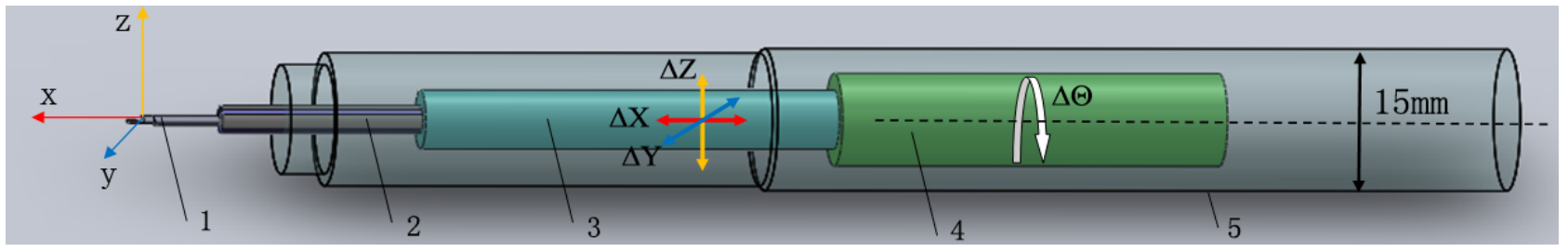

FIG. 1. Schematic drawing of the piezo-actuated nanomanipulation system. Labels: 1) TEM specimen and clamp. 2) Fine-actuation drive, 4-quadrant piezo tube. 3) Coarse-actuation drive (slipstick). 4) Rotary drive. 5) Hollow TEM specimen holder hull of standard diameter.

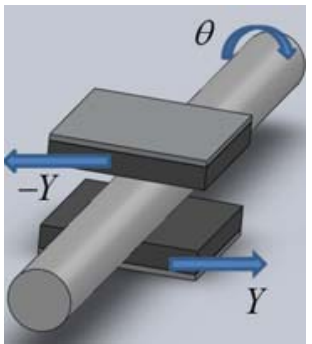

FIG. 2. Rotary drive mechanism. A shaft put inside a pair of piezos with opposite shearing directions.

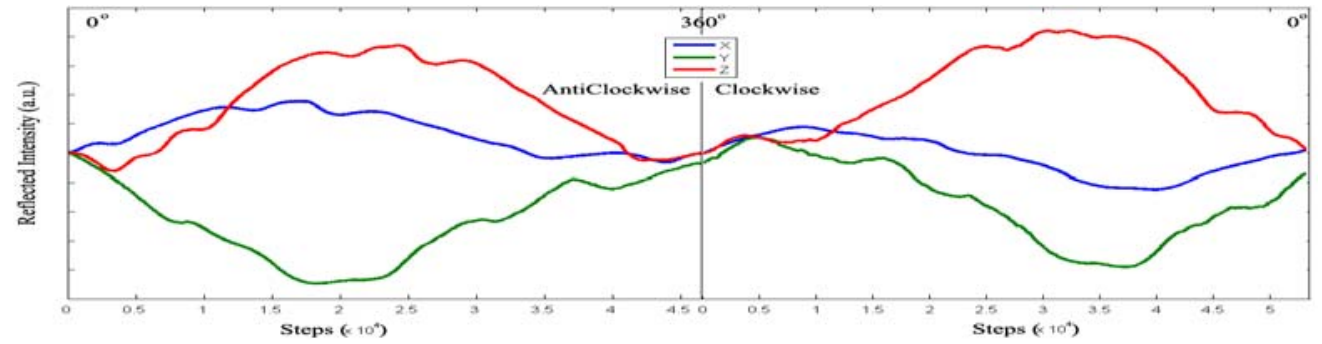

FIG. 3. Displacements of the specimen in 3 dimensions of 2 cycles of $360^{\circ}$ rotation (anticlockwise rotation on the left and clockwise one on the right). The reflected intensity was measured by fiber optical sensors of reflected light using a $2 \mathrm{~mm}$ polished sapphire rod as object.

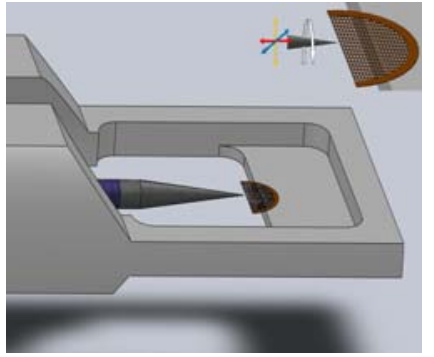

FIG. 4. A schematic diagram of the differential rotation between two specimen (using TEM goniometer for the fixed one and the piezo-rotation drive for the mobile one)

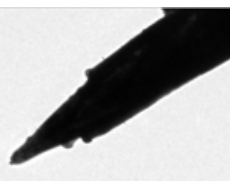

FIG. 5. A mobile W tip (mounted on the drives, left) is approaching a fixed $\mathrm{W}$ tip (mounted on the TEM sample holder, shown at the top right) shown at the bottom 